\title{
Peces de la plataforma continental de Michoacán y sus relaciones regionales en el Pacífico mexicano
}

\author{
Juan Madrid Vera 1, Arturo Ruiz Luna 2, Iván Rosado Bravo ${ }^{3}$ \\ 1 Instituto Nacional de la Pesca. Centro Regional de Investigaciones Pesqueras. Manzanillo, Col ., México. A.P. 591. e- \\ mail: jmv@ cucafera.icm.csic.es \\ 2 Centro de Investigación en Alimentación y Desarrollo. Unidad Mazatlán. Mazatlán, Sin., México. A.P. 711. e-mail: \\ arluna@servidor.unam.mx \\ 3 Departamento de Biología, Facultad de Ciencias, Universidad Nacional Autónoma de México C.P. 04510, México, D.F.
}

Recibido 5-VI-1997. Corregido 12-II-1998. Aceptado 23-II-1998.

\begin{abstract}
The ichthyofauna from the continental shelf of Michoacan (Mexico) was surveyed from June 1987 through June 1992 to produce a checklist of 257 species. The sample size and collection representativeness were validated by fitting a logistic model for the accumulative number of species $\left(S_{t}=298.86\left(1-e^{-0.122 t}\right)\right.$ ). In agreement with this model, $85 \%$ of the maximum forecast for cumulative species that can be caught under the same sampling conditions (298), have been identified. A probable scenario for the regional species interrelationships was obtained with a multivariate analysis that includes 1180 species (rows) belonging to 16 checklists (columns) of the northwestern Pacific Ocean ichthyofauna. From the analysis, the species composition on the Pacific shelf of Michoacan seems to be influenced by conditions similar to those of northern areas (lower Gulf of California) and not only by the physiography of the narrow coastal shelf of Michoacan.
\end{abstract}

Key words: Ichthyofauna, tropical Pacific, Mexico, regional relationships, checklist

El manejo de los recursos pesqueros marinos requiere del conocimiento de su diversidad así como de las relaciones regionales que se dan entre especies. Esto implica que deben conocerse los procesos a nivel de poblaciones y de comunidad, que permitan estimar en que forma son determinantes las conexiones entre especies y el ambiente para generar una mayor o menor biodiversidad y en consecuencia, cual es su efecto en la estabilidad de los sistemas.

En particular para el Pacífico nor-oriental, se cuenta con una importante documentación sobre la composición de la ictiofauna, aunque no sobre la naturaleza de las relaciones interes- pecíficas. Algunos de los trabajos más importantes para Norte y Centroamérica, si bien no son exclusivos para el Pacífico, son los realizados por Jordan y Evermann (1896-1900), quienes registraron aproximadamente 3200 especies de peces marinos y dulceacuícolas.

Para niveles de menor escala geográfica se cuenta con los trabajos de Miller y Lea (1972) y Horn y Allen (1978), quienes informan sobre un número cercano a las 600 especies para las costas de California. Concretamente para el Golfo de California, Present (1987) indica que el número de especies ícticas en la región puede ser ligeramente superior a 820. Por otra par- 
te van der Heiden y Findley (1988) publicaron una lista de 600 especies que habitan la parte sur del estado de Sinaloa, entre Piaxtla y Teacapán, en la entrada al Golfo de California.

Aunado a lo anterior, se cuenta con los trabajos de Castro-Aguirre (1978), YañezArancibia (1978), Alvarez et al. (1986) y Balart et al. (1992) para las especies que ingresan a los sistemas lagunar-estuarinos, a partir de los cuales se estima que existen aproximadamente 200 especies marinas que se desplazan hacia esos ambientes. Finalmente, en proximidad con el área de estudio se han llevado a cabo diversos trabajos, que indican que aproximadamente 400 especies ícticas han sido descritas para la región (Yañez-Arancibia 1978, Fuentes y Gaspar 1981, Amézcua 1985, Madrid et al. 1997).

A una escala mayor, en el área comprendida entre el Golfo de Papagayo, C.R. y las costas de California, E.U.A. $\left(9^{\circ}\right.$ a $\left.42^{\circ} \mathrm{N}\right)$, se estima un total cercano a 1200 especies marinas, agrupadas en 592 géneros y 4 clases, de acuerdo a lo reportado por Thomson et al. (1979), Acal y Arias (1990), Bianchi (1991), Rodríguez-Romero et al. (1994), de la CruzAgüero et al. (1994) y Abitia-Cárdenas et al. (1994), además de los autores anteriormente citados.

Lo anterior permite constatar la importancia de la región en términos de su diversidad, sin embargo, es evidente que existen vacíos de conocimiento que erróneamente podrían traducirse como variaciones de la diversidad. Por ello, es importante documentar la presencia de especies en las zonas menos conocidas, tal como es el caso de la mayor parte del Pacífico Mesoamericano, por lo que en este sentido el presente trabajo se ha centrado en la elaboración de una lista sistemática de los peces de la plataforma continental de Michoacán, en el Pacífico central mexicano y al mismo tiempo pretende coadyuvar a una mejor interpretación de sus relaciones regionales.

\section{MATERIALES Y METODOS}

Para cumplir con los objetivos señalados se realizaron dos estudios secuenciales. El primero de ellos concluye con la elaboración de un listado sistemático que permite contar con una visión local de la riqueza de especies. El segundo pretende visualizar a través del análisis de diversas listas de especies, las relaciones que éstas tienen dentro del Pacífico occidental.

Para la elaboración de la lista sistemática, se llevaron a cabo 18 muestreos (junio, agosto y diciembre/1987; enero, julio y octubre/1988; febrero, marzo y diciembre/1989; marzo, junio y diciembre/1990; julio, agosto, octubre y diciembre/1991; marzo y junio/1992), con duración aproximada de cinco días cada uno, durante la fase de luna nueva. Se realizaron en la costa de Michoacán $\left(18^{\circ} 02^{\prime}-18^{\circ} 10^{\prime} \mathrm{N} ; 102^{\circ}\right.$ $20^{\prime}-102^{\circ} 55^{\prime} \mathrm{W}$ ), en diversas localidades y campos pesqueros ubicados $70 \mathrm{~km}$ al NW del río Balsas. El radio de acción de las embarcaciones abarca una extensión aproximada de $50 \mathrm{~km}$ de línea de costa, con $1 \mathrm{~km}$ de ancho promedio.

Las muestras provienen de capturas comerciales de pesca artesanal y de pesca experimental, realizadas en la plataforma continental, a partir de la zona de intermareas y entre profundidades de 1 a $300 \mathrm{~m}$. Se incluyeron artes de pesca tales como línea y anzuelo, redes de tipo trasmallo y agalleras con luz de malla de 2.54 a $12.7 \mathrm{~cm}$ (1 a 5"), así como redes de cuchara y trampas cebadas que permitieron la captura de peces pelágicos, bénticos y demersales de diferentes ambientes (suaves, arenosos, pozas de marea y de comunidades coralinas o arrecife rocoso), considerándose un diseño de muestreo de tipo aleatorio estratificado.

Se analizaron en promedio 35 desembarques de la pesca comercial por muestreo, además de realizarse 11 censos en transectos paralelos a la línea de costa, cubriendo un área aproximada de $312 \mathrm{~m}^{2}$ (Rosado 1993). Así mismo, se efectuaron ocho recolectas en dos 
estaciones de la zona de intermareas (Villaseñor 1991). Se recolectó la mayoría de ejemplares considerados de escaso interés comercial, en tanto que para las especies de mayor valor se contabilizó el número de ejemplares. Se registró talla $(\mathrm{cm})$, peso $(\mathrm{g})$ y se revisaron las características merísticas y morfométricas de mayor importancia para la identificación. Para el listado final de especies se siguió la propuesta de Fischer et al. (1995), para los peces del Pacífico centro-oriental. Los ejemplares recolectados están depositados en el Departamento de Biología de la Facultad de Ciencias de la Universidad Nacional Autónoma de México.

El número acumulado de especies se ajustó a un modelo logístico $\left(S_{t}=A \cdot\left(1-e^{-b t}\right)\right)$, con objeto de determinar la representatividad de la muestra. St indica el número de especies que se incorporan por muestreo o unidad de tiempo $(t=$ cinco días), $b$ es la tasa instantánea de especies que se incorporan por vez primera a la lista sistemática en función del tiempo dedicado al muestreo y $A$ es el valor asintótico o máximo número de especies que podrían ser identificadas en el área de estudio a un nivel elevado de esfuerzo. Este valor está dado por la relación a/b (Colwell y Coddington 1994). Los valo es de los parámetros a y $b$, fueron encontrados por métodos no-lineales iterativos.

Para el análisis regional se integró una matriz de presencia-ausencia que incluye la lista de especies obtenida en este estudio y se agregan las listas para las áreas de California (Miller y Lea 1972, Horn y Allen 1978), las lagunas y esteros de Teacapán-Agua Brava (Alvarez et al. 1986) y de Guerrero (Yañez-Arancibia 1978); los ambientes rocosos y coralinos del Golfo de California (Thomson et al. 1979), el estuario del Río Balsas (Fuentes y Gaspar 1981), la plataforma continental del sur de Sinaloa (van der Heiden y Findley 1988) y de Nayarit a Guerrero (Amézcua 1985); las Bahías de Magdalena (de la Cruz-Aguero et al. 1994), Topolobampo, Ohuira y Santa María (Balart et al. 1992), bahía de Concepción (Rodríguez-Romero et al. 1994) y La Paz (Abi ia-
Cárdenas et al. 1994). Asimismo se incluyeron los listados de Acal y Arias (1990) para el Golfo de Tehuantepec y el de Bianchi (1991) desde el Golfo de Tehuantepec al Golfo de Papagayo en Costa Rica. Las sinonimias a nivel de género se resolvieron siguiendo a Eschmeyer (1990) y en el ámbito específico se consideraron algunas de las publicaciones ya citadas, además de Hildebrand (1946) y Monaco et al. (1992).

En función de lo anterior se obtuvo una matriz de 1180 especies x 16 listados (renglones $\mathrm{x}$ columnas). Aplicando métodos multivariados de clasificación, se generó un dendrograma empleando el cuadrado de la distancia euclideana como medida de similaridad y el método jerárquico WARD (Sneath y Sokal 1973), como algoritmo de agregación

\section{RESULTADOS}

Durante las 18 campañas se analizaron 22523 individuos, identificándose 257 especies pertenecientes a 157 géneros y 76 familias. Del total de especies, más del $33 \%$ se agruparon en cinco familias, siendo Carangidae la mejor representada, con 26 especies y siguiendo en ese orden Haemulidae (20), Sciaenidae (19), Carcharhinidae (11) y Serranidae (10). Las familias restantes están representadas por una a nueve especies. El arreglo sistemático se muestra en el Cuadro 1.

El número mí imo de individuos analizados por campaña de muestreo, con duración de cinco días, fue de 252 en octubre de 1991, con un máximo de 3409 en marzo de 1990 y un promedio de 1252 individuos por muestreo. El número de especies identificadas mostró un comportamiento lineal con respecto al tamaño de la muestra $\left(\mathrm{r}^{2}>0.60 ; P=95 \%\right)$, aunque para el número de especies que se incorporaban de manera acumulativa, la tendencia fue inicialmente en aumento y posteriormente, por arriba de las 250 especies, se estabilizó. 


\section{CUADRO 1}

Lista sistemática de peces de la plataforma continental de Michoacán, México

\section{ELASMOBRANCHII}

\section{ORDEN ORECTOLOBIFORMES}

Familia Ginglymostomatidae

1. Ginglymostoma cirratum (Bonnaterre, 1788) ORDEN LAMNIFORMES

Familia Lamnidae

2. Carcharodon carcharias (Linnaeus, 1758)

Familia Alopiidae

3. Alopias pelagicus Nakamura, 1935

4. A. superciliosus (Lowe, 1839)

ORDEN CARCHARHINIFORMES

Familia Triakidae

5. Mustelus californicus Gill, 1864

6. M. lunulatus Jordan y Gilbert, 1883

7. Triakis semifasciata Girard, 1854

Familia Carcharhinidae

8. Carcharhinus albimarginatus (Rüppell, 1837)

9. C. brachyurus (Günther, 1870)

1 0. C. falciformis (Bibron en Müller y Henle, 1839)

11. C. leucas (Valenciennes, 1839)

12. C. limbatus (Valenciennes, 1839)

13. C. obscurus (Lesueur, 1818)

14. C. porosus (Ranzani, 1839)

15. Galeocerdo cuvier (Peron y LeSueur, 1822)

16. Nasolamia velox (Gilbert en Jordan y Evermann, 1898)

17. Negaprion brevirostris (Poey, 1868)

18. Rhizoprionodon longurio (Jordan y Gilbert, 1882)

Familia Sphyrnidae

1. Sphyrna lewini (Griffith y Smith, 1834)

2. S. zygaena (Linnaeus, 1758)

\section{ORDEN RAJIFORMES}

Familia Rhinobatidae

3. Rhinobatos glaucostigma Jordan y Gilbert, 1884

4. R. productus Ayres, 1856

5. Zapteryx exasperata (Jordan y Gilbert, 1881)

ORDEN MYLIOBATIFORMES

Familia Dasyatidae

6. Dasyatis brevis (Garman, 1879)

Familia Myliobatidae

7. Aetobatus narinari (Euphrasen, 1790)

Familia Urolophidae

8. Urobatis halleri (Cooper, 1863)

9. Urotrygon chilensis (Günther, 1871)

10. U. munda Gill, 1863

\section{OSTEICHTHYES}

\section{ORDEN ELOPIFORMES}

Familia Elopidae

29. Elops affinis Regan, 1909

ORDEN ALBULIFORMES

Familia Albulidae

30. Albula nemoptera (Fowler, 1911)

31. A. cf. vulpes (Linnaeus, 1758)

\section{ORDEN ANGUILLIFORMES}

Familia Muraenidae

32. Gymnomuraena zebra (Shaw, 1797)

33. Gymnothorax castaneus (Jordan y Gilbert, 1882)

34. Muraena lentiginosa Jenyns, 1842

Familia Ophichthidae

35. Ophichthus triserialis (Kaup, 1856)

36. O. zophochir Jordan y Gilbert, 1881

Familia Congridae

37. Heteroconger digueti (Pellegrin, 1923)

ORDEN CLUPEIFORMES

Familia Clupeidae

38. Harengula thrissina (Jordan y Gilbert, 1882)

39. Opisthonema libertate (Günther, 1867)

Familia Pristigasteridae

40. Ilisha furthi (Steidachner, 1875)

Familia Engraulidae

41. Anchoa ischana (Jordan y Gilbert, 1882)

42. A. lucida (Jordan y Gilbert, 1882)

43. A. mundeola (Gilbert y Pierson, 1898)

44. A. scofieldi (Jordan y Culver, 1895)

45. Cetengraulis mysticetus (Günther, 1867)

ORDEN GONORHYNCHIFORMES

Familia Chanidae

46. Chanos chanos (Forsskål, 1775)

ORDEN SILURIFORMES

Familia Ariidae

47. Arius dasycephalus Günther, 1864

48. A. guatemalensis (Günther, 1864)

49. A. kessleri Steindachner, 1876

50. A. planiceps Steindachner, 1876

51. A. platypogon Günther, 1864

52. Bagre panamensis (Gill, 1863)

53. B. pinnimaculatus (Steindachner, 1876)

ORDEN AULOPIFORMES

Familia Synodontidae

54. Synodus scituliceps Jordan y Gilbert, 1881

ORDEN BATRACHOIDIFORMES

Familia Batrachoididae

5 5. Porichthys sp.

\section{ORDEN LOPHIIFORMES}

Familia Ogcocephalidae

56. Zalieutes elater (Jordan y Gilbert, 1882)

ORDEN GOBESOCIFORMES

Familia Gobiesocidae

57. Tomicodon boehlkei Briggs, 1955

58. T. zebra (Jordan y Gilbert, 1882)

ORDEN BELONIFORMES

Familia Belonidae

59. Platybelone argalus pterura (Osburn y Nichols, 1916)

60. Strongylura exilis (Girard, 1854)

61 . Tylosurus acus pacificus (Steindachner, 1876)

62. T. crocodilus fodiator Jordan y Gilbert, 1882

Familia Hemiramphidae

63. Hyporhamphus unifasciatus (Ranzani, 1842)

Familia Exocoetidae

64. Cypselurus callopterus (Günther, 1866)

65. Fodiator acutus rostratus (Günther, 1866) 


\section{ORDEN BERYCIFORMES}

Familia Holocentridae

6 6. Myripristis leiognathus Valenciennes, 1855

67 . Saragocentron suborbitalis (Gill, 1864)

\section{ORDEN SYNGNATHIFORMES}

Familia Fistulariidae

68 . Fistularia commersoni Rüppell, 1835

Familia Syngnathidae

69. Hippocampus ingens (Girard, 1859)

ORDEN SCORPAENIFORMES

Familia Scorpaenidae

7 0. Scorpaena histrio Jenyns, 1842

71. Scorpaena. $\mathrm{sp}$

Familia Triglidae

7 2. Bellator loxias Jordan en Gilbert 1896

73. Bellator xenisma (Jordan y Bollman, 1889)

74 . Prionotus ruscarius Gilbert y Starks, 1904

7 5. P. stephanophrys Lockington, 1881

ORDEN PERCIFORMES

Familia Centropomidae

76. Centropomus armatus Gill, 1863

77. C. medius Günther, 1864

78. C. nigrescens Günther, 1864

79. C. robalito Jordan y Gilbert, 1881

80 . C. viridis Lockington, 1887

Familia Serranidae

81 . Alphestes immaculatus Breder, 1936

82 . A. multiguttatus (Günther, 1867)

83. Cephalopholis panamensis (Steindachner, 1876)

84. Dermatolepis dermatolepis (Boulenger, 1895)

8 5. Diplectrum pacificum Meek y Hildebrand, 1925

8 6. Epinephelus acanthistius (Gilbert, 1892)

87. E. analogus Gill, 1864

8 8. E. labriformis (Jenyns, 1843)

89. Paranthias colonus (Valenciennes, 1855)

90. Rypticus bicolor Valenciennes, 1846

9 1. Rypticus sp.

92. Serranus psittacinus Valenciennes, 1855

Familia Priacanthidae

93. Heteropriacanthus cruentatus (Lacepède, 1801)

94. Pristigenys serrula (Gilbert, 1891)

Familia Apogonidae

95. Apogon retrosella (Gill, 1863)

Familia Echeneidae

96. Remora remora (Linnaeus, 1758)

Familia Carangidae

97. Alectis ciliaris (Bloch, 1788)

9 8. Caranx (Caranx) caballus Günther, 1868

99. C. (Caranx) caninus Günther, 1867

10 0.C. (Caranx) lugubris Poey, 1860

101.C. (Caranx) melampygus Cuvier, 1833

1 02 . C. (Caranx) otrynter Jordan y Gilbert, 1883

103.C.(Caranx) sexfasciatus Quoy y Gaimard, 1824

10 4.C. (Gnathanodon) speciosus (Forsskål, 1775)

105.C. (Carangoides) vinctus Jordan y Gilbert, 1882

106. Chloroscombrus orqueta Jordan y Gilbert, 1882

107 . Decapterus muroadsi (Temminck y Schlegel, 1844)

108 .Elagatis bipinnulata (Quoy y Gaimard, 1824)
109. Hemicaranx leucurus (Günther, 1864)

110. H. zelotes Gilbert, 1898

111 . Oligoplites altus (Günther, 1868)

112 . O. refulgens Gilbert y Starks, 1904

113 . O. saurus (Bloch y Schneider, 1801)

114 . Selar crumenophthalmus (Bloch, 1793)

115 . Selene brevoortii (Gill, 1863)

116 .S. oerstedii Lütken, 1880

117.S. peruviana (Guichenot, 1866)

11 8. Seriola peruana Steindachner, 1876

11 9. S. rivoliana Valenciennes, 1833

120. Trachinotus kennedyi Steindachner, 1875

121.T. paitensis Cuvier, 1832

122 .T. rhodopus Gill,1863

Familia Nematistiidae

123. Nematistius pectoralis Gill, 1862

Familia Coryphaenidae

12 4. Coryphaena hippurus Linnaeus, 1758

Familia Lutjanidae

125 . Hoplopagrus guntheri Gill, 1862

126 . Lutjanus argentiventris (Peters, 1869)

127 . L. colorado Jordan y Gilbert, 1882

128. L. guttatus (Steindachner, 1869)

129. L. inermis (Peters, 1869)

130 .L. novemfasciatus Gill, 1862

131 . L. peru (Nichols y Murphy, 1922)

Familia Lobotidae

132 . Lobotes surinamensis Bloch, 1790

Familia Gerreidae

133 . Diapterus aureolus (Jordan y Gilbert, 1882)

134 .D. peruvianus (Cuvier en Cuv. y Val., 1830)

135 . Eucinostomus currani Zahuranec en Yañez, 1980

136.E. gracilis (Gill, 1862)

137. Gerres cinereus (Walbaum, 1792)

Familia Haemulidae

138 . Anisotremus davidsoni (Steindachner, 1875)

139. A. dovi (Günther, 1864)

140. A. interruptus (Gill, 1862)

141. A. pacifici (Günther, 1864)

142. A. taeniatus (Gill, 1862)

143 . Haemulon flaviguttatum Gill, 1863

144 . H. maculicauda (Gill, 1863)

145 .H. scudderi Gill, 1863

146 . H. sexfasciatum Gill, 1863

147 . H. steindachneri (Jordan y Gilbert, 1882)

148 . Haemulopsis leuciscus (Günther, 1864)

149 . Orthopristis chalceus (Günther, 1864)

150. O. reddingi Jordan y Richardson, 1895

151 . Orthopristis sp.

152. Pomadasys axillaris (Steindachner, 1869)

153. P. bayanus (Jordan y Evermann, 1898)

15 4.P. macracanthus (Günther, 1864)

15 5. P. panamensis (Steindachner, 1875)

156.Xenichthys xanti Gill, 1863

157. Xenistius californiensis (Steindachner, 1875)

Familia Sparidae

158. Calamus brachysomus (Lockington, 1880)

Familia Sciaenidae 
159. Bairdiella armata Gill, 1863

160. B. ensifera (Jordan y Gilbert, 1862)

161 . B. incistia (Jordan y Gilbert, 1881)

162. Cynoscion reticulatus (Günther, 1864)

163. Elattarchus archidium (Jordan y Gilbert, 1882)

164 . Isopisthus remifer Jordan y Gilbert, 1881

165 . Larimus acclivis Jordan y Bristol, 1898

166.L. argenteus (Gill, 1863)

167.L. effulgens Gilbert, 1898

168 . Menticirrhus elongatus (Günther, 1864)

169.M. nasus (Günther, 1869)

170.M. panamensis (Steindachner, 1875)

171 . Micropogonias altipinnis (Günther, 1864)

172. Odontoscion xanthops Gilbert, 1898

173. Ophioscion imiceps (Jordan y Gilbert, 1881)

174. O. scierus (Jordan y Gilbert, 1884)

175. Pareques viola (Gilbert en Jordan y Evernnann, 1898)

176. Umbrina roncador Jordan y Gilbert, 1881

177. U. xanti Gill, 1862

Familia Mullidae

178. Mulloidichthys dentatus (Gill, 1863)

179. Pseudupeneus grandisquamis (Gill, 1863)

Familia Kyphosidae

180. Kyphosus analogus (Gill, 1863)

181. K. elegans (Peters, 1869)

182. Sectator ocyurus (Jordan y Gilbert, 1881)

Familia Ephippidae

1 83. Chaetodipterus zonatus Girard, 1858

184 . Parapsettus panamensis Steindachner, 1875

Familia Chaetodontidae

185. Chaetodon humeralis Günther, 1860

186 . Johnrandallia nigrirostris (Gill, 1862)

Familia Pomacanthidae

1 87. Holacanthuspasser Valenciennes en Cuv. y Val., 1846

1 8 8. Pomacanthus zonipectus (Gill, 1863)

Familia Pomacentridae

189. Abudefduf concolor (Gill, 1852)

190.A. troschelii (Gill, 1862)

191. Chromis atrilobata Gill, 1862

192. Microspathodon bairdii (Gill, 1863)

1 93.M. dorsalis (Gill, 1863)

194. Stegastes acapulcoensis (Fowler, 1944)

195.S. flavilatus (Gill, 1863)

196. S. leucorus (Gilbert, 1892)

1 97.S. rectifraenum (Gill, 1863)

Familia Cirrhitidae

198. Cirrhitichthys oxycephalus (Bleeker, 1855)

199. Cirrhitus rivulatus Valenciennes, 1855

Familia Mugilidae

200. Mugil cephalus Linnaeus, 1758

201 . M. curema Cuvier y Valenciennes, 1836

Familia Polynemidae

202. Polydactylus approximans (Lay y Bennett, 1839)

203.P. opercularis (Gill, 1863)

Familia Labridae

204 . Bodianus diplotaenia (Gill, 1863)

205 . Halichoeres chierchiae Caporiacco, 1947

206.H. dispilus (Günther, 1864)
207. H. nicholsi (Jordan y Gilbert, 1881)

208 .H. notospilus (Günther, 1864)

20 9. H. semicinctus (Ayres, 1859)

210 . Semicossyphus sp.

211 . Thalassoma lucasanum (Gill, 1863)

Familia Scaridae

212 . Scarus perrico Jordan y Gilbert, 1881

Familia Uranoscopidae

213 . Astroscopus zephyreus Gilbert y Starks en Gilbert, 1897

Familia Trypterygiidae

214 . Axoclinus carminalis (Jordan y Gilbert, 1882)

215 . Enneanectes sexmaculatus (Fowler, 1944)

Familia Clinidae (incl. Labrisomidae)

216 . Labrisomus striatus Hubbs, 1953

21 7. Malacoctenus ebisui Springer, 1959

21 8.M. hubbsi Springer, 1959

2 19.Malacoctenus sp.

Familia Chaenopsidae

22 0. Acanthemblemaria balanorum Brock, 1940

Familia Blenniidae

22 1. Ophioblennius steindachneri Jordan y Evermann, 1898

222 . Plagiotremus azaleus (Jordan y Bollman, 1890)

Familia Eleotrididae

223 . Gobiomorus maculatus (Günther, 1859)

224. Gobiomorus sp.

Familia Gobiidae

22 5. Bathygobius ramosus Ginsburg, 1947

226 . Bathygobius sp.

Familia Acanthuridae (incl. Zanclidae)

22 7. Acanthurus triostegus (Linnaeus, 1758)

228 . A. xanthopterus Valenciennes en Cuv. y Val., 1835

229. Acanthurus sp.

230 . Prionurus punctatus Gill, 1862

231 . Zanclus canescens (Linnaeus, 1758)

Familia Sphyraenidae

232 . Sphyraena ensis Jordan y Gilbert, 1882

233. S. lucasana Gill, 1863

Familia Istiophoridae

234 . Istiophorus platypterus (Shaw y Nodder, 1792)

Familia Scombridae

23 5. Auxis thazard (Lacepède, 1800)

236. Euthynnus lineatus Kishinouye, 1920

23 7. Katsuwonus pelamis (Linnaeus, 1758)

238 . Scomberomorus concolor (Lockington, 1879)

239. S. sierra Jordan y Starks, 1895

Familia Stromateidae

240 .Peprilus medius (Peters, 1869)

ORDEN PLEURONECTIFORMES

Familia Paralichthyidae

241 . Ancylopsetta dendritica Gilbert, 1890

242. Cyclopsetta querna (Jordan y Bollman, 1890)

243 . Etropus crossotus Jordan y Gilbert, 1882

244 . Hippoglossina tetrophthalma (Gilbert, 1890)

245.Paralichthys woolmani Jordan y Williams en

Gilbert, 1897

246 . Syacium ovale (Günther, 1864) 
Familia Bothidae

247 . Bothus constellatus (Jordan en Jordan y Goss, 1889) ORDEN TETRAODONTIFORMES

Familia Balistidae

248 . Balistes polylepis Steindachner, 1876

24 9. Pseudobalistes naufragium (Jordan y Starks, 1895)

250 . Sufflamen verres (Gilbert y Starks, 1904)

Familia Ostraciidae

251 . Ostracion meleagris Shaw, 1796

Familia Tetraodontidae

252. Arothron meleagris (Bloch y Schneider, 1801)

253. Canthigaster punctatissima (Günther, 1870)

254 . Sphoeroides annulatus (Jenyns, 1842)

255.S. lobatus (Steindachner, 1870)

Familia Diodontidae

256 . Diodon holocanthus Linnaeus, 1758

25 7. D. hystrix Linnaeus, 1758

El número acumulativo de especies obtuvo su mejor ajuste con la función $\mathrm{St}=298.86$ (1e-0.122t). Considerándose este modelo se proyectó para el doble de esfuerzo, equivalente a 90 días de muestreo adicionales, estimándose un total de 295 especies, lo que implica un incremento aproximado del $10 \%$ con respecto a las especies acumuladas al final de las $18 \mathrm{cam}$ pañas y un valor cercano al número asintótico de 298 especies (Fig. 1).

Con relación al análisis regional, a partir de los datos de presencia-ausencia de los 16

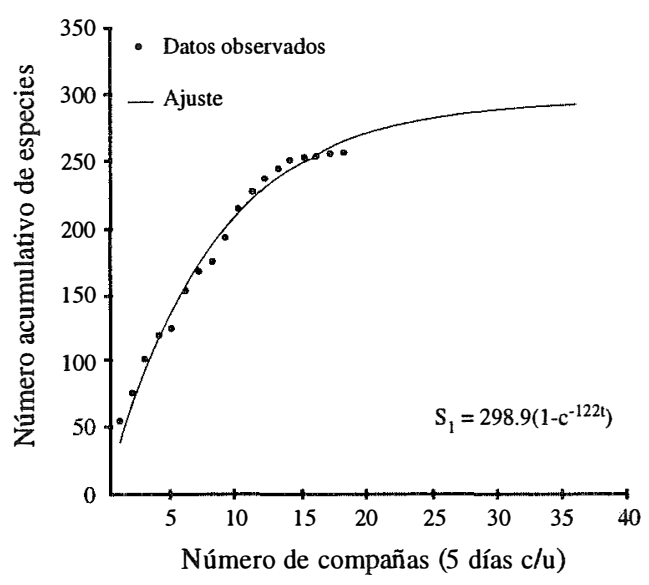

Fig. 1. Número acumulativo de especies de peces de la plataforma continental de Michoacán y curva de ajuste a un modelo logístico. listados de especies incluidos en el presente estudio, se generó una clasificación cuyo producto es un dendrograma, en el que se reconocen tres grupos principales. El primer grupo, que se destaca claramente de los otros dos grupos (Fig. 2-a), representa las listas de California (Miller y Lea 1972, Horn y Allen 1978). El grupo está asociado a peces de bahías y de estuarios, pelágicos y demersales. Los principales ambientes son macroalgales, macrofíticos, arena y comunidades coralígenas. El grupo está situado entre los $32^{\circ}$ y $42^{\circ}$ Norte, en la influencia de aguas frías y templadas de 15 a los $17^{\circ} \mathrm{C}$ de temperatura promedio en invierno y 15 a $19^{\circ} \mathrm{C}$ en verano.

Para el segundo grupo (Fig. 2-b) se separan principalmente los grupos asociados al Golfo de California o su área de influencia, incluyéndose la plataforma continental de Michoacán y de Colima. Predominan las especies de arrecifes y de fondos suaves. El cúmulo muestra la influencia subtropical entre los $18^{\circ}$ a los $26^{\circ} 55^{\prime}$ Norte, con temperaturas de entre 17 a $27^{\circ} \mathrm{C}$ en invierno y 19 a $29^{\circ} \mathrm{C}$ en verano. Finalmente se separó un tercer grupo (Fig. 2-c) que incluye básicamente especies registradas en lagunas costeras o zonas estuarinas, aunque es evidente una separación geográfica de las bahías Concepción y Magdalena en Baja California Sur, la fauna asociada a la zona comprendida entre Papagayo, Costa Rica y el golfo de Tehuantepec, propiamente Panámica y los ambientes estuarinos del Pacífico centro-norte del país. El grupo está situado entre los $9^{\circ} 00^{\prime}$ a los $26^{\circ} 05^{\prime}$ Norte, bajo la influencia tropical y subtropical, con temperaturas entre los 19 a los $28^{\circ} \mathrm{C}$ en invierno y de 28 a $29^{\circ} \mathrm{C}$ el verano.

\section{DISCUSION}

Es indudable que el número de especies listado para la región de estudio, la plataforma continental de Michoacán, pod ría incrementarse de manera significativa al ampliar el ámbito de muestreo, del que se excluyen, por limitaciones 


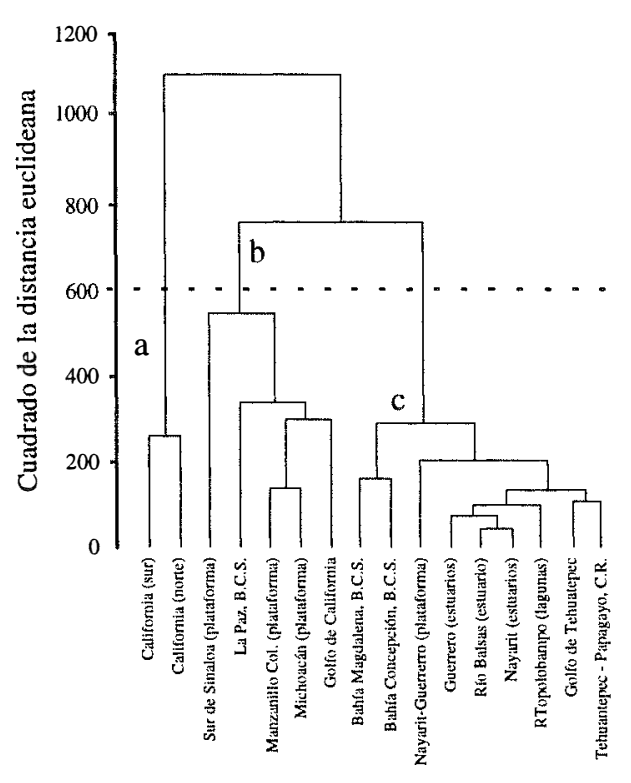

Fig. 2. Dendrograma de las regiones de afinidad ictiofaunística obtenidas con la aplicación de WARD como algoritmo de agregación y el cuadrado de la distancia euclideana como métrica.

de equipo, especies de peces pelágicos de media agua y de fondos fangosos y arenosos que pudieran ser capturados con redes de arrastre.

Sin embargo, bajo las condiciones en las que se desarrolló el presente trabajo, el tamaño de muestra parece suficiente para dar una visión cualitativa de la composición de la ictiofauna de la región. El resultado del ajuste de los datos al modelo logístico indica que al término del estudio, se contaba con mas del $85 \%$ del número de especies que teóricamente podrían ubicarse en los ambientes estudiados.

Las especies que aquí fueron identificadas superan de manera individual al número registrado por Yañez-Arancibia (1978), Fuentes y Gaspar (1981) y Amézcua (1985) en áreas adyacentes a la región de estudio. Estos trabajos informan en conjunto sobre un número cercano a 400 especies. Desde esa perspectiva, los resultados son indicativos de la representatividad del listado en términos de la riqueza o diversidad local de especies. Cabe destacarse que la identificación de algunas especies dentro del área de estudio representa una probable extensión de ámbito geográfico, misma que se está documentando en algunos de los casos para darle validez y rigor científico.

Esta probable extensión viene a validar la hipótesis que se plantea inicialmente, en el sentido que las variaciones en la diversidad para el centro del Pacífico mexicano en particular y del Pacífico mesoamericano en general son, sobre todo, vacios de información. El limitado número de estudios en la región central del Pacífico mexicano y la estrechez de su plataforma continental han propiciado que se equipare esta zona con un desierto, dada su limitada diversidad. Sin embargo, es posible que el incremento que se viene dando en el número y calidad de trabajos en esta región, demuestren en breve que la discontinuidad en la riqueza de especies no es un resultado exclusivo de las condiciones fisiográficas, sino también producto del abandono científico en el que se mantuvo esta región hasta fechas recientes.

Desde la perspectiva regional, es posible apreciar sesgos que muy probablemente se derivan de las características particulares de los muestreos (arte de pesca, área cubierta, época, tamaño de muestra), por lo que las relaciones regionales presentadas son sólo heurísticas. A pesar de ello y en términos generales, los vínculos determinados a través del análisis de clasificación son un reflejo de la posición climática y biogeográfica gradual de las costas mexicanas del Pacífico, tal como ha sido señalado por diversos autores que han estudiado la regionalización en el Pacífico y sus posibles conexiones ambientales (Ekman 1953, Briggs 1974, Hubbs 1974, Horn y Allen 1978, Allen y Smith, 1988).

Es importante resaltar asimismo que el análisis de cúmulos se hizo en función de las ausencias y presencias de especies en un área específica y no se usaron criterios cuantitativos, los que en su momento pudieran definir mas claramente el carácter de la relación entre 
las regiones incluidas en este estudio. Sin embargo, con la información disponible no es posible este tipo de planteamiento y deberán plantearse estrategias de formación de bases de datos que permitan realizar este tipo de análisis en el futuro.

Asimismo, el análisis jerárquico está realizado bajo el supuesto de que la clasificación sistemática Lineana es jerárquica y ésta es transitiva. Con relación a la influencia climática, la situación es diferente, pues se supone que su efecto es al azar sobre las poblaciones, sin embargo la dominancia de ciertas familias y géneros pueden permitir este tipo de análisis.

En suma, el análisis refleja la existencia de un importante número de especies para la costa michoacana, cuya riqueza parece estar determinada por condiciones similares a las que se presentan en la parte baja del Golfo de California y no solamente por los aspectos fisiográficos de la región, donde predomina una reducida plataforma continental y menor diversidad de ambientes, mismos que pudieran actuar como limitantes para el desarrollo de una mayor diversidad.

\section{AGRADECIMIENTOS}

La realización del presente estudio fue posible gracias a los apoyos ofrecidos por el Depto. de Biología de la Facultad de Ciencias de la Universidad Nacional Autónoma de México, el Instituto Nacional de la Pesca y el Consejo Nacional de Ciencia y Tecnología de México. Agradecemos a Albert van der Heiden la revisión del listado sistemático y a César Berlanga y Héctor Plascencia su revisión crítica.

\section{RESUMEN}

Como resultado de 18 campañas de muestreo llevadas a cabo desde junio de 1987 a junio de 1992 en la plataforma continental de Michoacán (México), se presenta un listado sistemático que incluye a 257 especies de peces.
Se determinó la representatividad de la muestra a nivel regional ajustando el número acumulativo de especies a una curva de tipo logístico. El resultado señala que se cuenta con mas del $85 \%$ del total de especies que teóricamente podrían capturarse en la región (298) en las mismas condiciones de muestreo. A partir de una matriz que incluye 1180 especies (renglones) correspondientes a 16 listados (columnas) elaborados por diversos autores para el Pacifico nor-oriental, se establecen las probables relaciones regionales por medio de un análisis jerárquico de clasificación. Se concluye que las condiciones ambientales con influencia en la boca del Golfo de California coadyuvan a determinar la riqueza de especies marinas en la plataforma continental de Michoacán.

\section{REFERENCIAS}

Abitia-Cárdenas, L.A., J. Rodríguez-Romero, F. Galván-Magaña, J. de la Cruz-Agüero \& H. Chávez-Ramos. 1994. Lista sistemática de la ictiofauna de Bahía de La Paz, Baja California Sur, México. Cienc. Mar. 20: 159-181.

Acal, D.E. \& A. Arias. 1990. Evaluación de los recursos demerso-pelágicos vulnerables a redes de arrastre de fondo en el sur del Pacífico de México. Cienc. Mar. 16: 93-129.

Alvarez, R.M., L.F. Amézcua \& A. Yañez-Arancibia. 1986. Ecología y estructura de las comunidades de peces en el sistema lagunar Teacapán-Agua Brava, Nayarit, México. Anal. Inst. Cienc. Mar y Limnol. UNAM. $13: 185-242$.

Allen, J.M. \& B.G. Smith. 1988. Atlas and zoogeography of common species in the Bering Sea and North Eastern Pacific. NOAA Tec. Rep. NMFS 66. 151 p.

Amézcua, L.F. 1985. Recursos potenciales de peces capturados con redes camaroneras en la costa del Pacífico de México, p. 39-94. In: Yañez-Arancibia, A. (ed.) Recursos pesqueros potenciales de México: La pesca acompañante del camarón. PUAL-ICMyL-INPUNAM. México. 743 p.

Balart, F.E., J.L. Castro-Aguirre \& R. Torres-Orozco. 1992. Ictiofauna de las bahías de Ohuira, Topolobampo y Santa María, Sinaloa, México. Invest. Mar. CICIMAR. 7: 93-103.

Bianchi, G. 1991 . Demersal assemblages of the continental shelf and slope edge between the Gulf of Tehuantepec (Mexico) and the Gulf of Papagayo (Costa Rica). Mar. Ecol. Prog. Ser. 73: 12 1-140.

Briggs, J.C. 1974. Marine Zoogeography. Mc. Graw Hill. Nueva York, 475 p. 
Castro-Aguirre, J.L. 1978. Catálogo sistemático de los peces marinos que penetran a las aguas continentales de México con aspectos zoogeográficos y ecológicos. Dep. Pesca. Dir. Gen. INP. Ser. Cient. 19. 298 p.

Colwell, R.K. \& A.J. Coddington. 1994. Estimating terrestrial biodiversity through extrapolation. Phil. Trans. Real Soc. London. 345: 101-118.

Cruz-Agüero J. de la, F. Galván-Magaña, L.A. Abitia-Cárdenas, J. Rodríguez-Romero \& F.J. Gutiérrez-Sánchez. 1994. Systematic list of marine fishes from Bahía Magdalena, Baja California Sur, Mexico. Cienc. Mar. 20: 17-31.

Chirichigno, N. 1982. Catálogo de especies marinas de interés económico actual o potencial para América Latina. Parte II. Pacífico centro y suroriental. FAO, Roma. $588 \mathrm{p}$.

Ekman, S. 1953. Zoogeography of the Sea. Sidgwick and Jackson. Londres. 417 p.

Eschmeyer, N.W. 1990. Catalogue of the genera of recent fishes. Calif. Ac. Sci. 697 p.

Fischer, W., F. Krupp, W. Schneider, C. Sommer, K.E. Carpenter \& V.H. Niem. 1995. Guía FAO para la identificación de especies para los fines de la pesc. Pacífico centro-oriental. FAO, Roma.. Vol. II y III. Parte 1 y 2: 647-1652.

Fuentes, M.P. \& M.T. Gaspar D. 1981. Aspectos biológicos y ecológicos de la ictiofauna de la desembocadura del río Balsas, Michoacán-Guerrero. Tesis Profesional. Fac. de Ciencias. UNAM. 192 p.

Heiden, A.M. van der \& L.T. Findley. 1988. Lista de los peces marinos del sur de Sinaloa, México. Anal. Inst. Cienc. Mar y Limnol. UNAM. 15, 209-224.

Hildebrand, F.S. 1946. A descriptive catalogue of the shore fishes of Peru. Smithsonian Inst. U.S. Nat. Mus. Bull.189. $530 \mathrm{p}$.

Horn, M.H. \& L.G. Allen. 1978. Adistributional analysis of California coastal marine fishes. J. Biogeogr. 5: 23-42.

Hubbs, C.L. 1974. Reviews and comments on the Marine zoogeography by John C. Briggs. Copeia 14: 1002-1005.
Jordan, D. S. \& Evermann, B.W. 1896, 1898 y 1900. The fishes of North and Middle America. Bull. U.S. Natl. Mus. $47.3313 \mathrm{p}$.

Madrid, V.J., P. Sánchez \& A. Ruiz L. (1997). Diversity and abundance of a tropical fishery on the Pacific shelf of Michoacán, Mẹ́xico. Est. Coast. Shelf Sci. 45: 485-495.

Miller, D.J. \& Lea, R. N. 1972. Guide to the coastal marine fishes of California. Dept. Fish and Game Bull. $157,249 \mathrm{p}$.

Monaco, E.M., A.T. Lowery \& L.R. Emmett. 1992. Assemblage of the U.S. west coast estuaries based on the distribution of fishes. J. Biogeogr. 19: 251-267.

Present, T. M. C. 1987. Genetic differentiation of disjunct Gulf of California and Pacific outer coast population of Hypsoblennius jenkisi. Copeia 4: 1010-1024.

Rodríguez-Romero, J., L.A. Abitia-Cárdenas, F. GalvánMagaña \& H. Chávez-Ramos. 1994. Composition, abundance and specific richness of fishes from Concepcion Bay, Baja California Sur, Mexico. Cienc. Mar. 20: $321-350$

Rosado B. H.I. 1993. Contribución al conocimiento de las comunidades de peces del arrecife rocoso aledaño al litoral de Caleta de Campos, Michoacán, México. Tesis Profesional. Fac. de Ciencias. UNAM. México. 58 p.

Sneath P.H.A. \& R.R. Sokal. 1973. Numerical taxonomy: the principles and practice of numerical classification. W. F. Freeman, San Francisco. 573 p.

Thomson, D.A., T.L. Findley \& N.A. Kerstiitch. 1979. Reef fishes of the sea of Cortez. The rocky-shore fishes of the Gulf of California. University of Arizona, Tucsón. $302 \mathrm{p}$.

Villaseñor A.H. 1991. Ecología de las comunidades de peces de la zona de intermarea de Caleta de Campos, Michoacán. Tesis Profesional. Fac. de Ciencias. UNAM. México. 76 p.

Yañez-Arancibia, A. 1978. Taxonomía, ecología y estructura de las comunidades de peces en lagunas costeras con bocas efímeras del Pacífico de México. Cent. Cien. Mar y Limnol. UNAM. Pub. esp. 2: 1-306. 